Research Article

\title{
Complex Factorizations of the Lucas Sequences via Matrix Methods
}

\author{
Honglin Wu \\ Department of Mathematics, Shanghai Business School, Shanghai 201400, China \\ Correspondence should be addressed to Honglin Wu; mathswu@163.com
}

Received 9 October 2013; Accepted 27 November 2013; Published 2 January 2014

Academic Editor: Mehmet Sezer

Copyright (C) 2014 Honglin Wu. This is an open access article distributed under the Creative Commons Attribution License, which permits unrestricted use, distribution, and reproduction in any medium, provided the original work is properly cited.

Firstly, we show a connection between the first Lucas sequence and the determinants of some tridiagonal matrices. Secondly, we derive the complex factorizations of the first Lucas sequence by computing those determinants with the help of Chebyshev polynomials of the second kind. Furthermore, we also obtain the complex factorizations of the second Lucas sequence by the similar matrix method using Chebyshev polynomials of the first kind.

\section{Introduction}

Given two nonzero integers $P$ and $Q$ satisfying $P^{2}-4 Q \neq 0$. The first Lucas sequence $\left\{U_{n}(P, Q)\right\}_{n \geq 0}$ and the second Lucas sequence $\left\{V_{n}(P, Q)\right\}_{n \geq 0}$ are defined by the recurrence relations

$$
\begin{gathered}
U_{0}(P, Q)=0, \\
U_{1}(P, Q)=1, \\
U_{n}(P, Q)=P U_{n-1}(P, Q)-Q U_{n-2}(P, Q), \quad n \geq 2, \\
V_{0}(P, Q)=2, \\
V_{1}(P, Q)=P, \\
V_{n}(P, Q)=P V_{n-1}(P, Q)-Q V_{n-2}(P, Q), \quad n \geq 2,
\end{gathered}
$$

respectively.

By assigning $P$ and $Q$ some special values, we will see some well-known Lucas sequences, which are important historically and for their own sake. The numbers $\left\{U_{n}(1,-1)\right\}_{n>0}$ are called the Fibonacci numbers while the numbers $\left\{V_{n}(1,-1)\right\}_{n \geq 0}$ are called the Lucas numbers, the numbers $\left\{U_{n}(2,-1)\right\}_{n \geq 0}$ and $\left\{V_{n}(2,-1)\right\}_{n \geq 0}$ are the Pell numbers and the Pell-Lucas numbers, $\left\{U_{n}(1,-2)\right\}_{n \geq 0}$ and $\left\{V_{n}(1,-2)\right\}_{n \geq 0}$ are Jacobsthal numbers and Jacobsthal-Lucas numbers, respectively, $\left\{U_{n}(3,2)\right\}_{n \geq 0}$ are Mersenne numbers, and so on.
There is a long tradition of using matrix methods to study Lucas sequences [2-5]. In 2003, Cahill et al. obtained complex factorizations for Fibonacci numbers and Lucas numbers by using the determinants of two slightly different sequences of tridiagonal matrices [2]. They used the $n \times n$ tridiagonal matrix $M(n)$ with entries $m_{k, k}=1(1 \leq k \leq n)$, and $m_{k-1, k}=m_{k, k-1}=\mathrm{i}(2 \leq k \leq n)$ to prove that

$$
F_{n}=\prod_{k=1}^{n-1}\left(1-2 \mathrm{i} \cos \frac{\pi k}{n}\right), \quad n \geq 2
$$

where $F_{n}$ is the $n$th Fibonacci number and $\mathrm{i}=\sqrt{-1}$, and also used the $n \times n$ tridiagonal matrix $S(n)$ with entries $s_{1,1}=1 / 2$, $s_{k, k}=1(2 \leq k \leq n)$ and $s_{k-1, k}=s_{k, k-1}=\mathrm{i}(2 \leq k \leq n)$ to prove that

$$
L_{n}=\prod_{k=1}^{n}\left(1-2 \mathrm{i} \cos \frac{\pi(k-(1 / 2))}{n}\right), \quad n \geq 1,
$$

where $L_{n}$ is the $n$th Lucas number and $\mathrm{i}=\sqrt{-1}$. In 2011, Burcu Bozkurt et al. obtained the complex factorization of the second Lucas sequence $\left\{V_{n}(P,-1)\right\}_{n>0}[4]$ :

$$
V_{n}=\prod_{k=1}^{n}\left(P-2 \mathrm{i} \cos \frac{\pi(k-(1 / 2))}{n}\right), \quad n \geq 1,
$$

by using the $n \times n$ tridiagonal matrix $D(n)$ with entries $d_{1,1}=$ $P / 2, d_{k, k}=P(2 \leq k \leq n)$, and $d_{k-1, k}=d_{k, k-1}=\mathrm{i}(2 \leq k \leq n)$, where $\mathrm{i}=\sqrt{-1}$. 
In this study, number theory and linear algebra (with the help of orthogonal polynomials) are similarly intertwined to yield the complex factorizations of the first and second Lucas sequences. later.

Now we give the following lemma which will be needed

Lemma $1([2])$. Let $\{H(n), n=1,2, \ldots\}$ be a sequence of tridiagonal matrices of the form

$$
H(n)=\left(\begin{array}{ccccc}
h_{1,1} & h_{1,2} & & & \\
h_{2,1} & h_{2,2} & h_{2,3} & & \\
& h_{3,2} & h_{3,3} & \ddots & \\
& & \ddots & \ddots & h_{n-1, n} \\
& & & & h_{n, n-1} \\
& & & h_{n, n}
\end{array}\right) .
$$

Then the successive determinants of $H(n)$ are given by the recursive formula:

$$
\begin{aligned}
|H(1)|= & h_{1,1}, \quad|H(2)|=h_{1,1} h_{2,2}-h_{1,2} h_{2,1}, \\
|H(n)|= & h_{n, n}|H(n-1)| \\
& -h_{n-1, n} h_{n, n-1}|H(n-2)|, \quad n \geq 3 .
\end{aligned}
$$

\section{Complex Factorizations of the First Lucas Sequence}

First of all, we introduce the tridiagonal matrix sequence $\{A(n), n=1,2, \ldots\}$ and express the first Lucas sequence $U_{n}(P, Q)$ by the determinant of $A(n-1)$ for $n \geq 2$. Then we use this connection to prove the explicit formula for $U_{n}(P, Q)$ which is a generalization of the Binet Form for $U_{n}(P,-1)$.

Theorem 2. Let $\{A(n), n=1,2, \ldots\}$ be a sequence of tridiagonal matrices of the form

$$
A(n)=\left(\begin{array}{llllll}
P & \alpha & & & \\
\beta & P & \alpha & & \\
& \beta & P & \ddots & \\
& & \ddots & \ddots & \alpha \\
& & & & & \\
& & & & P
\end{array}\right)
$$

with $\alpha \beta=Q$, where $P$ and $Q$ are nonzero integers satisfying $P^{2}-4 Q \neq 0$. Then

$$
\begin{gathered}
U_{n}(P, Q)=|A(n-1)| \quad \text { for } n \geq 2, \\
U_{n}(P, Q)=\frac{a^{n}-b^{n}}{a-b} \quad \text { for } n \geq 0
\end{gathered}
$$

where $|\cdot|$ denotes the determinant $a$ and $b$ are roots of $x^{2}-P x+$ $Q=0$.
Proof. According to Lemma 1, successive determinants of $A(n)$ are given by the recursive formula:

$$
\begin{gathered}
|A(1)|=P, \quad|A(2)|=P^{2}-Q, \\
|A(n)|=P|A(n-1)|-Q|A(n-2)|, \quad n \geq 3 .
\end{gathered}
$$

Clearly, this is also the first Lucas sequence, starting with $U_{2}(P, Q)$. Therefore,

$$
U_{n}(P, Q)=|A(n-1)| \quad \text { for } n \geq 2 .
$$

Since $a$ and $b$ are roots of $x^{2}-P x+Q=0$,

$$
a+b=P, \quad a b=Q .
$$

Note that $U_{0}(P, Q)=0=\left(a^{0}-b^{0}\right) /(a-b)$ and $U_{1}(P, Q)=$ $1=\left(a^{1}-b^{1}\right) /(a-b)$. In order to prove $(10)$, it is sufficient to prove

$$
|A(n-1)|=\frac{a^{n}-b^{n}}{a-b} \quad \text { for } n \geq 2
$$

by (9). Now we prove (14) by induction on $n$. When $n=2,3$, we have

$$
\begin{gathered}
|A(1)|=P=a+b=\frac{a^{2}-b^{2}}{a-b}, \\
|A(2)|=P^{2}-Q=(a+b)^{2}-a b=a^{2}+a b+b^{2}=\frac{a^{3}-b^{3}}{a-b} .
\end{gathered}
$$

Next we assume (14) holds for $3 \leq n \leq k$. Then when $n=k+1$, we have

$$
\begin{aligned}
|A(k)| & =P|A(k-1)|-Q|A(k-2)| \\
& =(a+b)|A(k-1)|-a b|A(k-2)| \\
& =(a+b) \frac{a^{k}-b^{k}}{a-b}-a b \frac{a^{k-1}-b^{k-1}}{a-b} \\
& =\frac{a^{k+1}-a b^{k}+a^{k} b-b^{k+1}-a^{k} b+a b^{k}}{a-b} \\
& =\frac{a^{k+1}-b^{k+1}}{a-b} .
\end{aligned}
$$

Hence, (14) is proved.

Now we give two complex factorizations and one explicit formula of the first Lucas sequence by using the determinants of tridiagonal matrices and Chebyshev polynomials of the second kind.

Theorem 3. The first Lucas sequence $\left\{U_{n}(P, Q)\right\}_{n \geq 0}$ satisfies

$$
\begin{gathered}
U_{n}(P, Q)=\prod_{k=1}^{n-1}\left(P+2 \sqrt{Q} \cos \frac{k \pi}{n}\right), \quad n \geq 2, \\
U_{n}(P, Q)=\prod_{k=1}^{n-1}\left(P-2 \sqrt{Q} \cos \frac{k \pi}{n}\right), \quad n \geq 2, \quad \\
U_{n}(P, Q)=(\sqrt{Q})^{n-1} \frac{\sin (n \arccos (P / 2 \sqrt{Q}))}{\sin (\arccos (P / 2 \sqrt{Q}))}, \quad n \geq 1,
\end{gathered}
$$

where $\sqrt{\mathrm{Q}} \equiv \sqrt{-\mathrm{Q}} \mathrm{i}$ and $\mathrm{i}=\sqrt{-1}$ if $\mathrm{Q}$ is a negative integer. 
Proof. Choosing $\alpha=\beta=\sqrt{Q}$ in $\{A(n), n=1,2, \ldots\}$, where $\sqrt{Q}$ represents $\sqrt{-Q} i$ when $Q$ is negative, we obtain a sequence of tridiagonal matrices $\{B(n), n=1,2, \ldots\}$ of the form

$$
B(n)=\left(\begin{array}{ccccc}
P & \sqrt{Q} & & & \\
\sqrt{Q} & P & \sqrt{Q} & & \\
& \sqrt{Q} & P & \ddots & \\
& & \ddots & \ddots & \sqrt{Q} \\
& & & \sqrt{Q} & P
\end{array}\right) .
$$

Then

$$
U_{n+1}(P, Q)=|B(n)| \quad \text { for } n \geq 1
$$

by Theorem 2 . Considering that the determinant of a matrix can be found by taking the product of its eigenvalues, we will compute the spectrum of $B(n)$ in order to find an alternate formulation for $|B(n)|$.

We now introduce another sequence of tridiagonal matrices $\{G(n), n=1,2, \ldots\}$, where $G(n)$ is the $n \times n$ tridiagonal matrix with $g_{k, k}=0(1 \leq k \leq n)$ and $g_{k-1, k}=g_{k, k-1}=1(2 \leq$ $k \leq n)$. That is,

$$
G(n)=\left(\begin{array}{ccccc}
0 & 1 & & & \\
1 & 0 & 1 & & \\
& 1 & 0 & \ddots & \\
& & \ddots & \ddots & 1 \\
& & & 1 & 0
\end{array}\right) .
$$

Note that $B(n)=P I_{n}+\sqrt{Q} G(n)$, where $I_{n}$ is the $n \times n$ identity matrix. Let $\lambda_{k}, k=1,2, \ldots, n$, be the eigenvalues of $G(n)$ with associated eigenvectors $x_{k}$. Then for each $1 \leq j \leq n$, we have

$$
\begin{aligned}
B(n) x_{j} & =\left(P I_{n}+\sqrt{\mathrm{Q}} G(n)\right) x_{j} \\
& =P I_{n} x_{j}+\sqrt{\mathrm{Q}} G(n) x_{j} \\
& =P x_{j}+\sqrt{\mathrm{Q}} \lambda_{j} x_{j} \\
& =\left(P+\sqrt{\mathrm{Q}} \lambda_{j}\right) x_{j} .
\end{aligned}
$$

Therefore, $P+\sqrt{\mathrm{Q}} \lambda_{k}, k=1,2, \ldots, n$ are the eigenvalues of $B(n)$. Hence,

$$
|B(n)|=\prod_{k=1}^{n}\left(P+\sqrt{Q} \lambda_{k}\right), \quad n \geq 1 .
$$

Next we compute $\lambda_{k}$ 's by recalling that each $\lambda_{k}$ is a zero of the characteristic polynomial $p_{n}(\lambda)=\left|\lambda I_{n}-G(n)\right|$. Notice that

$$
\lambda I_{n}-G(n)=\left(\begin{array}{ccccc}
\lambda & -1 & & & \\
-1 & \lambda & -1 & & \\
& & & & \\
& -1 & \lambda & \ddots & \\
& & \ddots & \ddots & -1 \\
& & & -1 & \lambda
\end{array}\right) \text {; }
$$

we use Lemma 1 to obtain a recursive formula for the characteristic polynomials of $\{G(n), n=1,2, \ldots\}$ :

$$
\begin{gathered}
p_{1}(\lambda)=\lambda, \quad p_{2}(\lambda)=\lambda^{2}-1, \\
p_{n}(\lambda)=\lambda p_{n-1}(\lambda)-p_{n-2}(\lambda), \quad n \geq 3 .
\end{gathered}
$$

This family of characteristic polynomials can be transformed into another family $\left\{U_{n}(x), n=1,2, \ldots\right\}$ by the transformation $\lambda \equiv 2 x$ as follows:

$$
\begin{gathered}
U_{1}(x)=2 x, \quad U_{2}(x)=4 x^{2}-1, \\
U_{n}(x)=2 x U_{n-1}(x)-U_{n-2}(x), \quad n \geq 3,
\end{gathered}
$$

and this family $\left\{U_{n}(x), n=1,2, \ldots\right\}$ is the set of Chebyshev polynomials of the second kind. It is well known [6] that defining $x \equiv \cos \theta$ allows the Chebyshev polynomials of the second kind to be written as follows:

$$
U_{n}(x)=\frac{\sin [(n+1) \theta]}{\sin \theta} .
$$

From (28), we can easily see that the roots of $U_{n}(x)=0$ are $\theta_{k}=k \pi /(n+1), k=1,2, \ldots, n$, or equally, $x_{k}=\cos \theta_{k}=$ $\cos (k \pi /(n+1)), k=1,2, \ldots, n$. So we get the eigenvalues of $G(n)$ as follows:

$$
\lambda_{k}=2 \cos \frac{k \pi}{n+1}, \quad k=1,2, \ldots, n .
$$

Combining (21), (24), and (29), we finally have

$$
U_{n+1}(P, Q)=\prod_{k=1}^{n}\left(P+2 \sqrt{Q} \cos \frac{k \pi}{n+1}\right), \quad n \geq 1,
$$

which is equivalent to (17). Moreover, by taking $k=n+1-t$, we get

$$
\begin{aligned}
U_{n+1}(P, Q) & =\prod_{t=1}^{n}\left(P+2 \sqrt{Q} \cos \left(\pi-\frac{t \pi}{n+1}\right)\right) \\
& =\prod_{t=1}^{n}\left(P-2 \sqrt{Q} \cos \frac{t \pi}{n+1}\right), \quad n \geq 1,
\end{aligned}
$$

which is identical to (18). 
From (25), we can think of Chebyshev polynomials of the second kind as being generated by determinants of successive matrices of the form

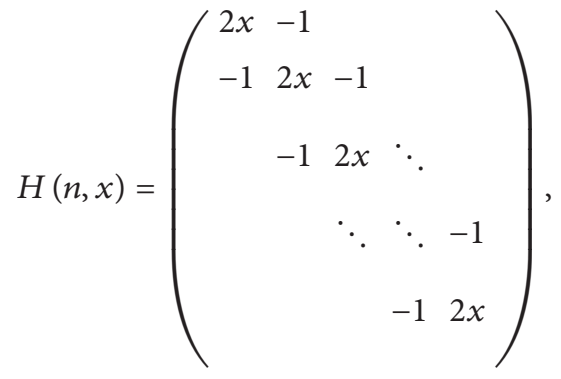

where $H(n, x)$ is $n \times n$. Note that $B(n)=-\sqrt{Q} H(n,-P / 2 \sqrt{Q})$. So we obtain

$$
|B(n)|=(-\sqrt{Q})^{n}\left|H\left(n,-\frac{P}{2 \sqrt{Q}}\right)\right|=(-\sqrt{Q})^{n} U_{n}\left(-\frac{P}{2 \sqrt{Q}}\right) .
$$

Since the Chebyshev polynomial of the second kind $U_{n}(x)$ is an even or odd function, involving only even or odd powers of $x$, as $n$ is even or odd, the right side of (33) can be represented as

$$
(-\sqrt{\mathrm{Q}})^{n} U_{n}\left(-\frac{P}{2 \sqrt{\mathrm{Q}}}\right)=(\sqrt{\mathrm{Q}})^{n} U_{n}\left(\frac{P}{2 \sqrt{\mathrm{Q}}}\right) .
$$

Combining (21), (33), (34), and (28), we derive

$$
U_{n+1}(P, Q)=(\sqrt{Q})^{n} \frac{\sin ((n+1) \arccos (P / 2 \sqrt{Q}))}{\sin (\arccos (P / 2 \sqrt{Q}))}, \quad n \geq 1 \text {, }
$$

which is identical to (19) since it also holds for $n=0$ :

$$
U_{1}(P, Q)=1=(\sqrt{Q})^{0} \frac{\sin (\arccos (P / 2 \sqrt{Q}))}{\sin (\arccos (P / 2 \sqrt{Q}))} .
$$

Remark 4. For $P=1, Q=-1,(18)$ and (19) are exactly the formulas for Fibonacci numbers in [2].

\section{Complex Factorizations of the Second Lucas Sequence}

The complex factorization of $\left\{V_{n}(P,-1)\right\}_{n \geq 0}$ was derived in [4], and we generalize the formula to $\left\{V_{n}(P, Q)\right\}_{n \geq 0}$ for any integer $Q$ in the following theorem.

Theorem 5. The second Lucas sequence $\left\{V_{n}(P, Q)\right\}_{n \geq 0}$ satisfies

$$
\begin{aligned}
& V_{n}(P, Q)=\prod_{k=1}^{n}\left(P+2 \sqrt{Q} \cos \frac{(k-(1 / 2)) \pi}{n}\right), \quad n \geq 1, \\
& V_{n}(P, Q)=\prod_{k=1}^{n}\left(P-2 \sqrt{Q} \cos \frac{(k-(1 / 2)) \pi}{n}\right), \quad n \geq 1,
\end{aligned}
$$

$$
V_{n}(P, Q)=2(\sqrt{Q})^{n} \cos \left(n \arccos \left(\frac{P}{2 \sqrt{Q}}\right)\right), \quad n \geq 1,
$$

where $\sqrt{\mathrm{Q}} \equiv \sqrt{-\mathrm{Q}} \mathrm{i}$ and $\mathrm{i}=\sqrt{-1}$ if $\mathrm{Q}$ is a negative integer.

Proof. In order to obtain (37), we introduce a sequence of tridiagonal matrices $\{F(n), n=1,2, \ldots\}$ of the form

$$
F(n)=\left(\begin{array}{ccccc}
\frac{P}{2} & \sqrt{Q} & & & \\
\sqrt{Q} & P & \sqrt{Q} & & \\
& \sqrt{Q} & P & \ddots & \\
& & \ddots & \ddots & \sqrt{Q} \\
& & & \sqrt{Q} & P
\end{array}\right) \text {, }
$$

where $\sqrt{Q}$ represents $\sqrt{-Q} i$ when $Q$ is negative. Then

$$
V_{n}(P, Q)=2|F(n)|
$$

by Theorem 1 of [4]. Unlike the derivation in Theorem 3, we will not compute the spectrum of $F(n)$ directly. Instead, since $\left|I_{n}+e_{1} e_{1}^{T}\right|=2$, we have

$$
|F(n)|=\frac{1}{2}\left|\left(I_{n}+e_{1} e_{1}^{T}\right) F(n)\right|,
$$

where $I_{n}$ is the $n \times n$ identity matrix and $e_{j}$ is the $j$ th column of $I_{n}$. Moreover, we can express the right-hand side of (42) as follows:

$$
\frac{1}{2}\left|\left(I_{n}+e_{1} e_{1}^{T}\right) F(n)\right|=\frac{1}{2}\left|P I_{n}+\sqrt{Q}\left(G(n)+e_{1} e_{2}^{T}\right)\right|,
$$

where $G(n)$ is the $n \times n$ matrix defined in Theorem 3 . Let $\mu_{k}, k=1,2, \ldots, n$ be the eigenvalues of $G(n)+e_{1} e_{2}^{T}$ with associated eigenvectors $y_{k}$. Then for each $1 \leq j \leq n$, we get

$$
\begin{aligned}
\left(P I_{n}\right. & \left.+\sqrt{Q}\left(G(n)+e_{1} e_{2}^{T}\right)\right) y_{j} \\
& =P I_{n} y_{j}+\sqrt{Q}\left(G(n)+e_{1} e_{2}^{T}\right) y_{j} \\
& =P y_{j}+\sqrt{Q} \mu_{j} y_{j} \\
& =\left(P+\sqrt{Q} \mu_{j}\right) y_{j} .
\end{aligned}
$$

Therefore, $P+\sqrt{Q} \mu_{k}, k=1,2, \ldots, n$, are the eigenvalues of $P I_{n}+\sqrt{Q}\left(G(n)+e_{1} e_{2}^{T}\right)$. Hence,

$$
\left|\left(I_{n}+e_{1} e_{1}^{T}\right) F(n)\right|=\prod_{k=1}^{n}\left(P+\sqrt{Q} \mu_{k}\right), \quad n \geq 1 .
$$


Next we compute $\mu_{k}$ 's by recalling that each $\mu_{k}$ is a zero of the characteristic polynomial $q_{n}(\mu)=\mid \mu I_{n}-(G(n)+$ $\left.e_{1} e_{2}^{T}\right) \mid$. Since $\left|\left(I_{n}-(1 / 2) e_{1} e_{1}^{T}\right)\right|=1 / 2$, we can represent the characteristic polynomial as follows:

$$
q_{n}(\mu)=2\left|\left(I_{n}-\frac{1}{2} e_{1} e_{1}^{T}\right)\left(\mu I_{n}-\left(G(n)+e_{1} e_{2}^{T}\right)\right)\right| .
$$

Notice that

$$
\begin{array}{r}
\left(I_{n}-\frac{1}{2} e_{1} e_{1}^{T}\right)\left(\mu I_{n}-\left(G(n)+e_{1} e_{2}^{T}\right)\right) \\
=\left(\begin{array}{cccccc}
\frac{\mu}{2} & -1 & & & \\
-1 & \mu & -1 & & \\
& = & \mu & & \\
& & & \ddots & \ddots & \\
& & & & -1 & \mu
\end{array}\right) .
\end{array}
$$

We use Lemma 1 to obtain a recursive formula for the characteristic polynomials:

$$
\begin{gathered}
q_{1}(\mu)=\frac{\mu}{2}, \quad q_{2}(\mu)=\frac{\mu^{2}}{2}-1, \\
q_{n}(\mu)=\mu q_{n-1}(\mu)-q_{n-2}(\mu), \quad n \geq 3 .
\end{gathered}
$$

This family of characteristic polynomials can be transformed into another family $\left\{T_{n}(x), n=1,2, \ldots\right\}$ by taking $\mu=2 x$ :

$$
\begin{gathered}
T_{1}(x)=x, \quad T_{2}(x)=2 x^{2}-1, \\
T_{n}(x)=2 x T_{n-1}(x)-T_{n-2}(x), \quad n \geq 3 .
\end{gathered}
$$

In fact, this family $\left\{T_{n}(x), n=1,2, \ldots\right\}$ is the set of Chebyshev polynomials of the first kind. It is well known [6] that defining $x \equiv \cos \theta$ allows the Chebyshev polynomials of the first kind to be written as

$$
T_{n}(x)=\cos n \theta .
$$

It is easy to see that the roots of $T_{n}(x)=0$ are given by $\theta_{k}=$ $(k-(1 / 2)) \pi / n, k=1,2, \ldots, n$, or $x_{k}=\cos \theta_{k}=\cos (k-$ $(1 / 2)) \pi / n, k=1,2, \ldots, n$. Thus, we derive

$$
\mu_{k}=2 \cos \frac{(k-(1 / 2)) \pi}{n}, \quad k=1,2, \ldots, n .
$$

Combining (41), (42), (45), and (51), we finally have

$$
V_{n}(P, Q)=\prod_{k=1}^{n}\left(P+2 \sqrt{Q} \cos \frac{(k-(1 / 2)) \pi}{n}\right), \quad n \geq 1 .
$$

Furthermore, by taking $k=n+1-t$, we obtain

$$
\begin{aligned}
V_{n}(P, Q) & =\prod_{t=1}^{n}\left(P+2 \sqrt{Q} \cos \left(\pi-\frac{(t-(1 / 2)) \pi}{n}\right)\right) \\
& =\prod_{t=1}^{n}\left(P-2 \sqrt{Q} \cos \frac{(t-(1 / 2)) \pi}{n}\right), \quad n \geq 1 .
\end{aligned}
$$

Equations (37) and (38) are proved.
Now we think of Chebyshev polynomials of the first kind from (47) as being generated by determinants of successive matrices of the form

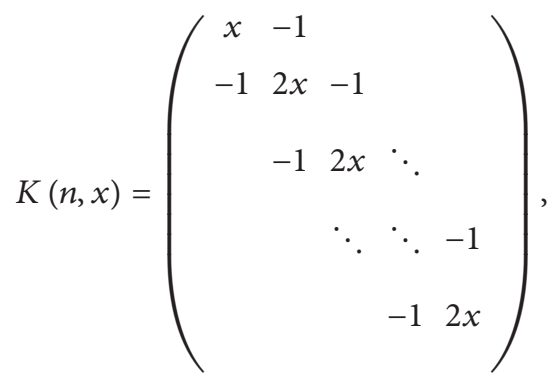

where $K(n, x)$ is $n \times n$. Note that $F(n)=-\sqrt{Q} K(n,-P / 2 \sqrt{Q})$. So we get

$$
|F(n)|=(-\sqrt{Q})^{n}\left|K\left(n,-\frac{P}{2 \sqrt{Q}}\right)\right|=(-\sqrt{Q})^{n} T_{n}\left(-\frac{P}{2 \sqrt{Q}}\right) .
$$

Since the Chebyshev polynomial of the first kind $T_{n}(x)$ is an even or odd function, involving only even or odd powers of $x$, according as $n$ is even or odd, the right side of (55) can be represented as

$$
(-\sqrt{\mathrm{Q}})^{n} T_{n}\left(-\frac{P}{2 \sqrt{\mathrm{Q}}}\right)=(\sqrt{\mathrm{Q}})^{n} T_{n}\left(\frac{P}{2 \sqrt{\mathrm{Q}}}\right) .
$$

Combining (41), (55), (56), and (50), we derive

$$
V_{n}(P, Q)=2(\sqrt{Q})^{n} \cos \left(n \arccos \left(\frac{P}{2 \sqrt{Q}}\right)\right), \quad n \geq 1 .
$$

Equation (39) is completed.

Remark 6. For $P=1, Q=-1,(38)$ and (39) are exactly the formulas for Lucas numbers in [2].

Remark 7. For $Q=-1,(38)$ and (39) are exactly the formulas for $\left\{V_{n}(P,-1)\right\}$ in $[4]$.

\section{Examples}

Finally, we give some examples.

Example 1. Choosing $P=2, Q=-1$, we can get the complex factorization of Pell numbers by (17). Let $P_{n}$ be the $n$th Pell number and $\mathrm{i}=\sqrt{-1}$. Then the fourth Pell number is

$$
\begin{aligned}
P_{4}= & U_{4}(2,-1)=\prod_{k=1}^{3}\left(2+2 i \cos \frac{k \pi}{4}\right) \\
= & \left(2+2 i \cos \frac{\pi}{4}\right)\left(2+2 i \cos \frac{2 \pi}{4}\right) \\
& \times\left(2+2 i \cos \frac{3 \pi}{4}\right) \\
= & (2+\sqrt{2} i)(2+0)(2-\sqrt{2} i) \\
= & 12 .
\end{aligned}
$$


Example 2. Choosing $P=1, Q=-2$, we can get the complex factorization of Jacobsthal numbers by (18). Let $J_{n}$ be the $n$th Jacobsthal number and $\mathrm{i}=\sqrt{-1}$. Then the third Jacobsthal number is

$$
\begin{aligned}
J_{3} & =U_{3}(1,-2)=\prod_{k=1}^{2}\left(1-2 \sqrt{2} i \cos \frac{k \pi}{3}\right) \\
& =\left(1-2 \sqrt{2} i \cos \frac{\pi}{3}\right)\left(1-2 \sqrt{2} i \cos \frac{2 \pi}{3}\right) \\
& =(1-\sqrt{2} i)(1+\sqrt{2} i) \\
& =3 .
\end{aligned}
$$

Example 3. Choosing $P=2, Q=-1$, we can get the complex factorization of Pell-Lucas numbers by (37). Let $p_{n}$ be the $n$th Pell-Lucas number and $\mathrm{i}=\sqrt{-1}$. Then the third Pell-Lucas number is

$$
\begin{aligned}
p_{3} & =V_{3}(2,-1)=\prod_{k=1}^{3}\left(2+2 i \cos \frac{(k-(1 / 2)) \pi}{3}\right) \\
& =\left(2+2 i \cos \frac{\pi}{6}\right)\left(2+2 i \cos \frac{3 \pi}{6}\right)\left(2+2 i \cos \frac{5 \pi}{6}\right) \\
& =(2+\sqrt{3} i)(2+0)(2-\sqrt{3} i) \\
& =14 .
\end{aligned}
$$

Example 4. Choosing $P=1, Q=-2$, we can get the complex factorization of Jacobsthal-Lucas numbers by (38). Let $j_{n}$ be the $n$th Jacobsthal-Lucas number and $\mathrm{i}=\sqrt{-1}$. Then the third Jacobsthal-Lucas number is

$$
\begin{aligned}
j_{3}= & V_{3}(1,-2)=\prod_{k=1}^{3}\left(1-2 \sqrt{2} i \cos \frac{(k-(1 / 2)) \pi}{3}\right) \\
= & \left(1-2 \sqrt{2} i \cos \frac{\pi}{6}\right)\left(1-2 \sqrt{2} i \cos \frac{3 \pi}{6}\right) \\
& \times\left(1-2 \sqrt{2} i \cos \frac{5 \pi}{6}\right) \\
= & (1-\sqrt{6} i)(1-0)(1+\sqrt{6} i) \\
= & 7 .
\end{aligned}
$$

\section{Conclusion}

We use the tridiagonal matrices $B(n)$ and $F(n)$ to obtain the complex factorizations of the first and second Lucas sequences, respectively. It is possible to find other suitable matrices for different factorizations of Lucas sequences.

\section{Conflict of Interests}

The author declares that there is no conflict of interests regarding the publication of this article.

\section{Acknowledgments}

The author's research is supported by Excellent Young Teacher Foundation of Shanghai (sxy10004) and Foundation of Shanghai Business School (A-0201-00-050-205). The author also appreciates the editors and the anonymous referees for their helpful suggestions and comments.

\section{References}

[1] P. Ribenboim, My Numbers, My Friends, Popular Lectures on Number Theory, Springer, Berlin, Germany, 2000.

[2] N. D. Cahill, J. R. D’Errico, and J. P. Spence, "Complex factorizations of the Fibonacci and Lucas numbers," The Fibonacci Quarterly, vol. 41, no. 1, pp. 13-19, 2003.

[3] N. D. Cahill and D. A. Narayan, "Fibonacci and Lucas numbers as tridiagonal matrix determinants," The Fibonacci Quarterly, vol. 42, no. 3, pp. 216-221, 2004.

[4] Ş. Burcu Bozkurt, F. Yilmaz, and D. Bozkurt, "On the complex factorization of the Lucas sequence," Applied Mathematics Letters, vol. 24, no. 8, pp. 1317-1321, 2011.

[5] J. Feng, "Fibonacci identities via the determinant of tridiagonal matrix," Applied Mathematics and Computation, vol. 217, no. 12, pp. 5978-5981, 2011.

[6] J. C. Mason and D. C. Handscomb, Chebyshev Polynomials, CRC Press, 2003. 


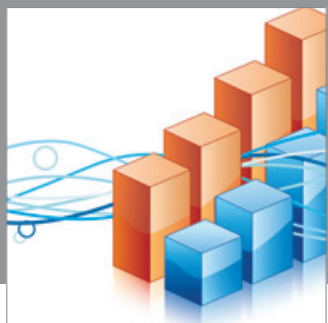

Advances in

Operations Research

mansans

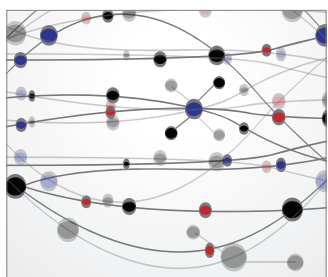

The Scientific World Journal
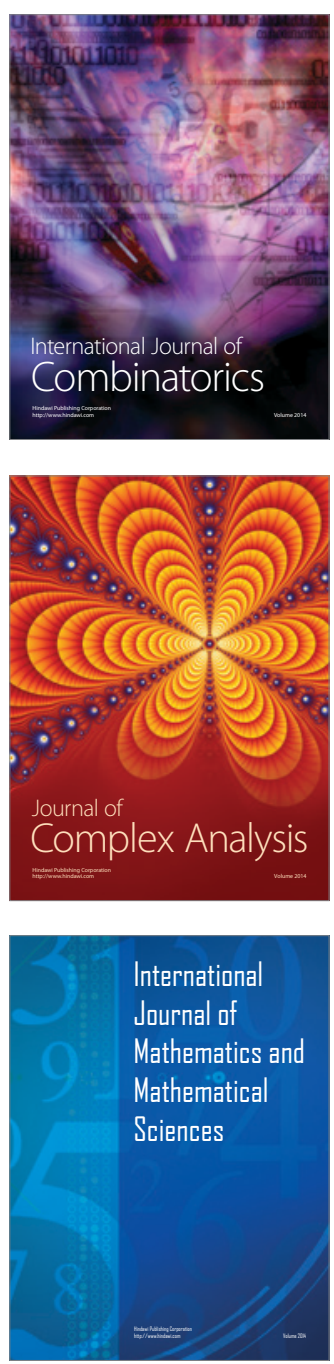
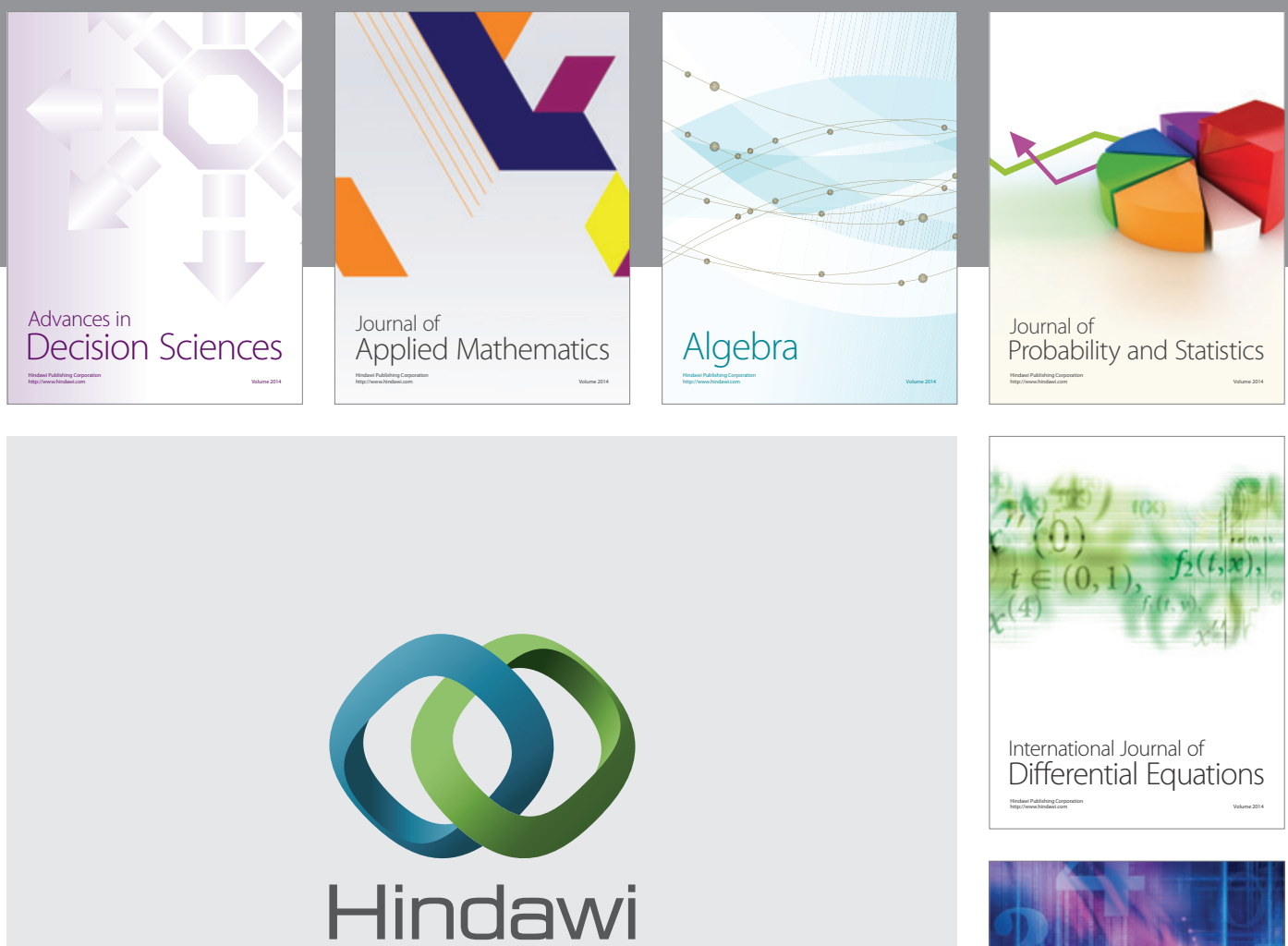

Submit your manuscripts at http://www.hindawi.com
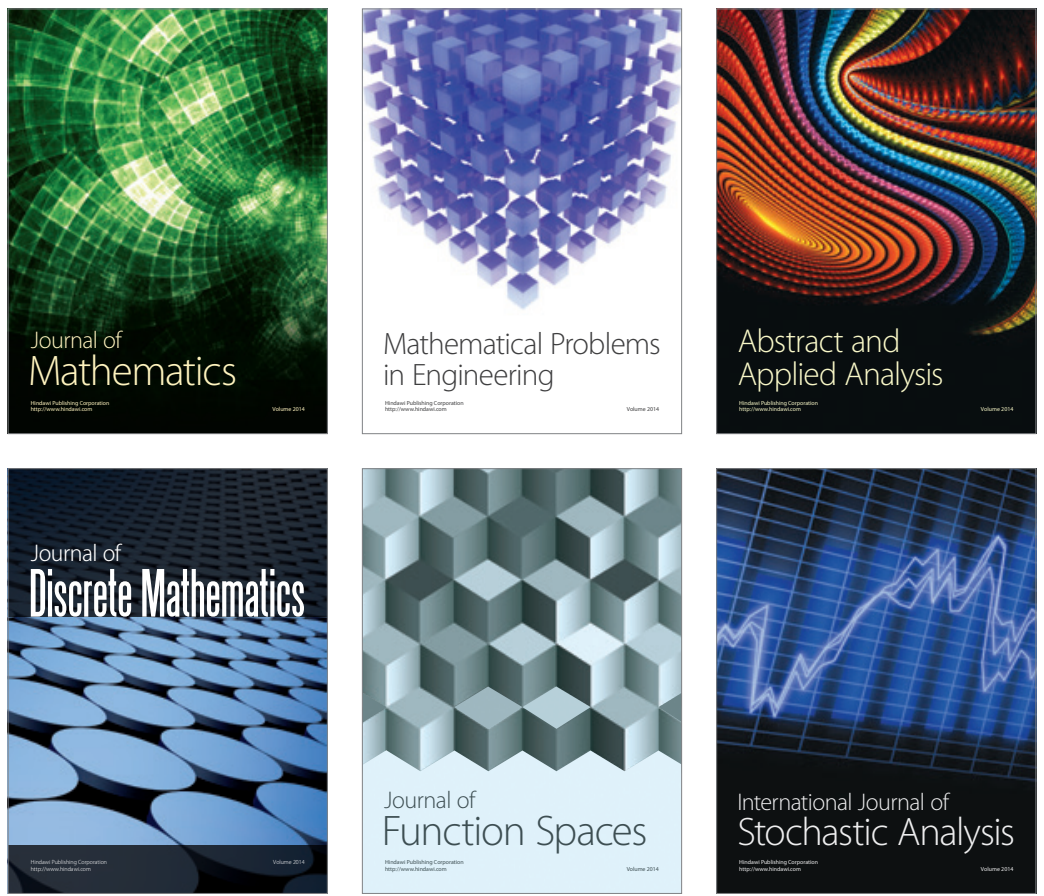

Journal of

Function Spaces

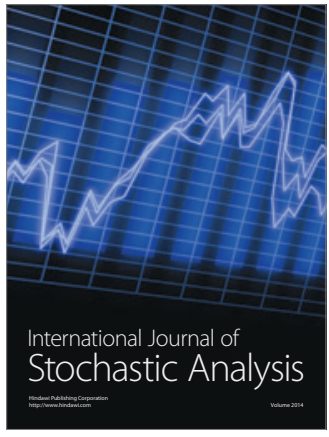

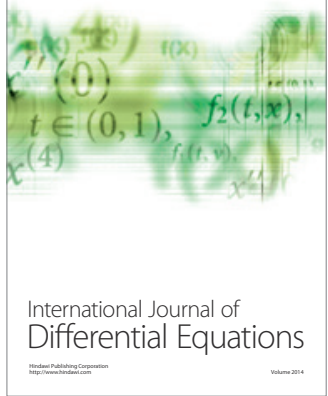
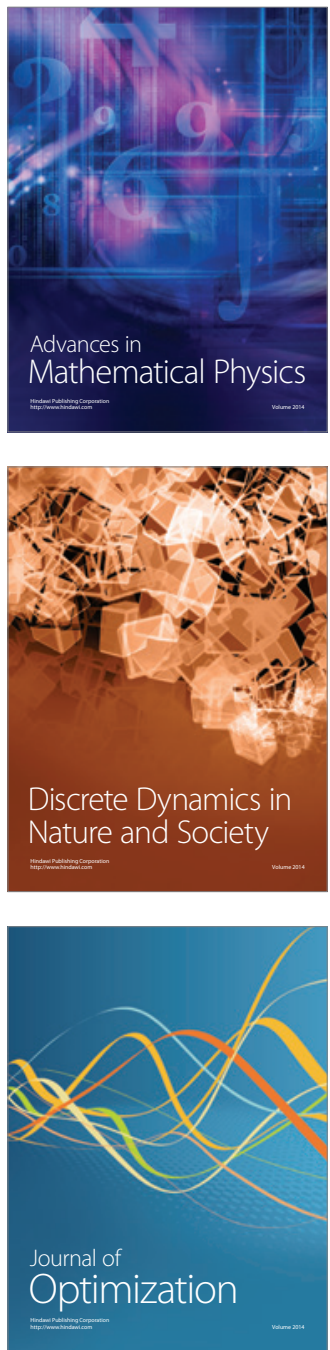